\title{
SUR LES VERS PARASITES DES CHIMÈRES
}

\author{
Par J. S. RUSZKOWSKI
}

L'un des poissons les plus intéressants, malheureusement pas assez connu au point de vue biologique, est certainement Chimaera monstrosa L.

On le capture sur les différents points de la Méditerranée et de l'Atlantique; on le trouve cependant le plus souvent sur les côtes norvégiennes. J'ai eu l'occasion d'en avoir un assez grand nombre, pendant mes deux séjours successifs à la Station biologique de Herdla (Bergens Museums Biologiske Stasjon) et par conséquent d'étudier leurs parasites avec beaucoup de précision. Je me suis spécialement attaché à l'examen de Gyrocotyle urna, parasite de la valvule spirale, appartenant à la sous-classe des Cestodaria. Une note a déjà été publié par moi à ce sujet (1). Dans cette étude, je me propose de décrire, si possible, tous les vers parasites des chimères et de donner en même temps quelques renseignements concernant leur statistique.

J'ai étudié les parasites des chimères dans la seconde moitié du mois de juillet et dans la première moitié d'août 1927, ainsi qu'à partir de la fin de juin jusqu'au mois de septembre 1929. J'ai examiné dans la première période trois chimères, dans la seconde, 90 poissons. Ces poissons étaient capturés au moyen d'une ligne de fond. Cette ligne, longue d'environ de $1.000 \mathrm{~m}$., munie de hameçons au nombre de 500 à 600 , avec des amorces constituées par des fragments de hareng, était posée sur un fond d'environ 200 à $300 \mathrm{~m}$. et laissée là pendant une ou deux heures. On prenait de cette façon, le plus souvent, Spinax niger Bon., ensuite les poissons appartenant aux Rajidés, Pristiurus catulus (Gunnerus), Squalus acanthias L., les genres Gadus, Molva et de temps en temps Chimaera monstrosa.

Il était difficile de se procurer par ce moyen de pêche les exemplaires jeunes des chimères. On capturait toujours les individus adultes de $55 \mathrm{~cm}$. ̀̀ 1 mètre de longueur. Le rapport des mâles aux

(1) Ruszkowski (J. S.). - Etudes sur le cycle évolutif et sur la structure des Cestodes de mer, $2^{e}$ partie, Sur les larves de Gyrocotyle urna (Gr. et Wagen). Bull. Ac. Pol. d. Sc., Cracovie, 1932.

Annales de Parasttologie, $\mathrm{T}$. XII, $\mathrm{N}^{\circ} 6 .-1^{\mathrm{er}}$ novembre 1934 , p. $482-491$. 
femelles était de $30: 63$, donc presque égal au rapport $1: 2$ (1). A peu près le tiers du matériel provenait des pêches effectuées par le bateau «Herman Friele », appartenant à la Station. C'était vraiment le matériel le plus précieux, puisque déjà 2 à 3 heures après la pêche, quelquefois même plus tôt, je pouvais procéder à l'examen des poissons, les chimères, et surtout les parasites, étant parfaitement vivants. Par contre, les poissons fournis par les pêcheurs étaient généralement morts et souvent dans un état de macération si avancée (surtout les branchies) qu'à part le matériel le plus important, tel que les œufs de Gyrocotyle urna, ils ne pouvaient absolument pas servir à l'étude. D'autre part, il m'a été impossible de faire la statistique des formes larvaires assez énigmatiques, trouvées dans le cœur et les branchies.

Voici les vers parasites dont j'ai pu relever la présence dans les chimères.

\section{Cestodaria}

Gyrocotyle urna Gr. et Wag.

Trematoda

Octobothrium leptogaster S. Leuck. Macraspis elegans Olss.
Calicotyle kroyeri Dies.

Lebouria sp.

Distomum sp.

Metacercarix dans le tube digestif. Miracidia? dans le cœur et les branchies.

Les trois premières espèces ont été trouvées dans les chimères par les différents auteurs, également en dehors de la Norvège ; les deux suivantes sont hébergées aussi par d'autres hôtes; enfin les formes larvaires n'ont pas été décrites jusqu’à présent.

Je dois remarquer que, malgré l'abondance du matériel, il m'a été impossible de trouver dans les chimères le trématode Distomum veliporum Creplin, signalé pour les chimères de la Méditerranée par Wagener et Parona. Par contre, je les ai trouvées quelquefois dans différentes raies, conformément aux observations de B. Olsson sur la présence de ces parasites dans les poissons de Norvège.

Les poissons paraissent constituer l'aliment principal des chimères, mais cette question n'est malheureusement pas suffisamment étudiée. En dehors de débris de poissons je trouvai souvent, dans le tube digestif, des gammaridés, des isopodes, des décapodes (par ex. Munida sp. et autres), parfois des polychètes, des coquilles de mollusques, des piquants d'échinodermes et des débris de petits céphalopodes.

(1) Etant donnée l'insuffisance de nos connaissances relatives à l'embryologie des chimères, il ne sera peut-être pas inutile de signaler que j'ai trouvé dans les oviductes de ces poissons un œuf le 31-7-29 et deux oufs le 3-8-29. 


\section{Gyrocotyle urna (Grube et Wagener)}

Gyrocotyle urna représente le parasite le plus fréquent des chimères. Sur les 93 exemplaires de ce poisson examinés, un seul n'en était pas porteur. Cependant, même ce cas unique ne peut pas être considéré comme négatif, étant donné le fait maintes fois constaté que G. urna quitte son hôte par le cloaque, de suite après sa mort. J'ai eu l'occasion d'observer ces parasites après leur sortie, parfaitement vivants et remuant activement. Il est possible que, même dans le cas négatif précité, la sortie du ver avait pu m'échapper.

Le nombre de G. urna, dans une seule chimère, peut s'élever, d'après les auteurs, à 7 exemplaires. Personnellement je n'ai jamais constaté un nombre si élevé. Le plus souvent, dans $72 \mathrm{p}$. cent des cas, il y en avait 2, plus rarement 1 ou 3 exemplaires ; trois fois, il y en avait 4 et une seule fois, le 25-8-29, j'ai trouvé le maximum de 5 parasites. J'ai des raisons de croire que les chimères capturées en pleine mer sont plus infestées que celles qui proviennent des fiords.

A côté des individus adultes, dont la taille variait de 2 à $6 \mathrm{~mm} ., 5$, j'ai trouvé aussi 4 larves longues de 3 à $10 \mathrm{~mm}$., 7, toutes pourvues encore de crochets embryonnaires. Ces crochets, disposés dans la région pourvue de rosette, m'ont permis d'établir d'une façon définitive que c'est précisément cette région qui constitue l'extrémité postérieure. L'orientation du parasite a été basée jusqu'à présent sur les caractères anatomiques, tirés des individus adultes. Il en résultait deux opinions diamétralement opposées. Les partisans des deux camps adverses s'efforçaient de découvrir de nouveaux caractères à l'appui de leurs thèses. Je me suis spécialement occupé de cette question dans mon étude parue en 1931-1932.

J'ai trouvé plusieurs fois les procercoïdes dans le parenchyme de G. urna adultes et une seule fois dans celui de la larve. Ils représentaient sans aucun doute les larves de G. urna, étant donnée la présence de 10 crochets dont ils étaient munis. Comment ont-ils pu y pénétrer ? je l'ignore ; cependant j'estime que leur présence dans le parenchyme ne constitue pas le chaînon normal du cycle évolutif de cet intéressant parasite.

J'ai trouvé dans mon matériel un cas anormal, dont il est rarement question dans les descriptions des parasites marins. Il s'agit du dédoublement de la région postérieure du parasite (fig. 1). Ce dédoublement, tout en s'étendant assez loin vers l'utérus, n'avait pas entraîné de changements importants dans l'appareil reproducteur, à l'exception de la diminution de taille d'une moitié des ovaires 
et leur asymétrie. Par contre, la rosette présentait des modifications plus importantes, puisqu'elle était divisée en deux parties inégales. Les plis latéraux qui renferment les vitellaria ont subi

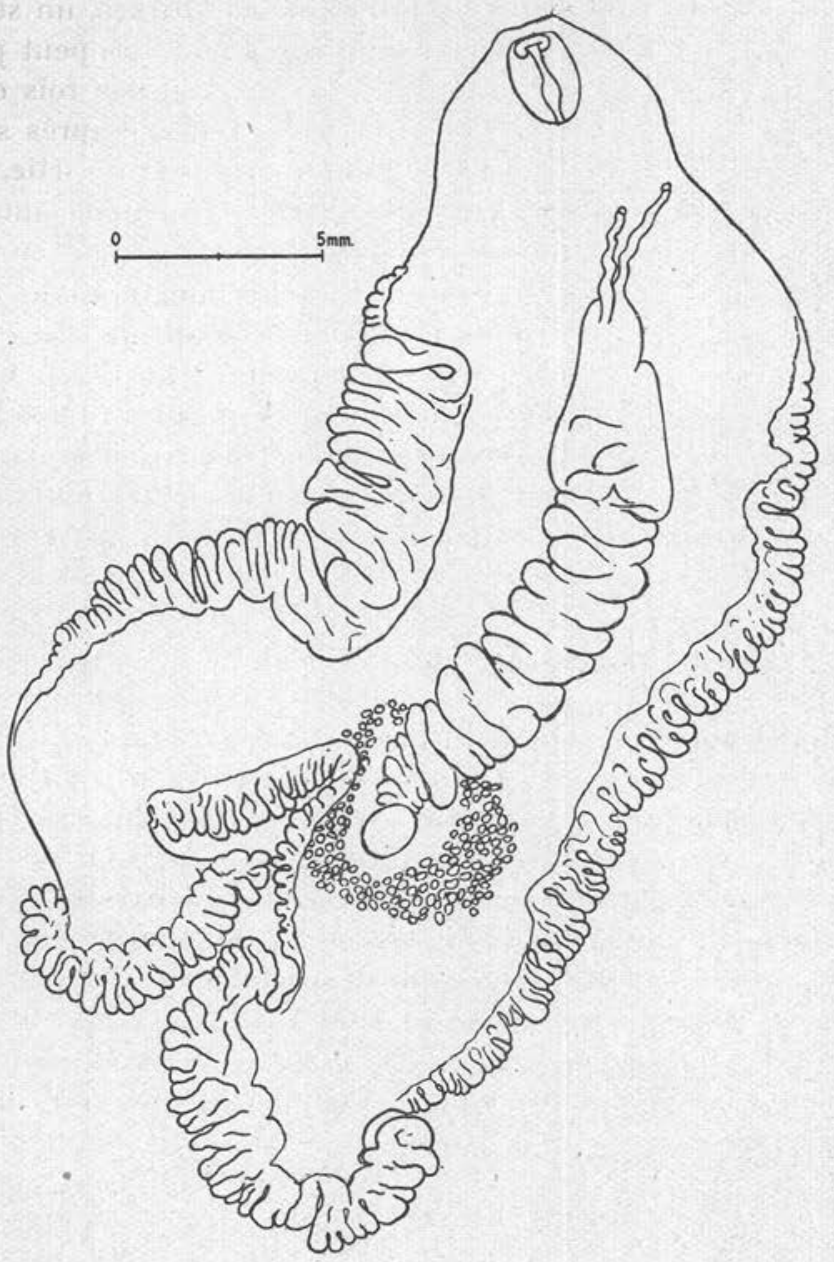

Fig. 1. - Gyrocotyle urna anormal.

aussi quelques changements ; ils apparaissent nettement au milieu du dédoublement et manquent complètement à la face externe de la plus petite partie.

Je n'ai pas constaté d'autres modifications, au moins sur les préparations in toto. 


\section{Octobothrium leptogaster F. S. Leuckart 1842}

C'est un des trématodes le plus fréquent, puisque le nombre des chimères infestées par lui atteignait 63 p. cent.

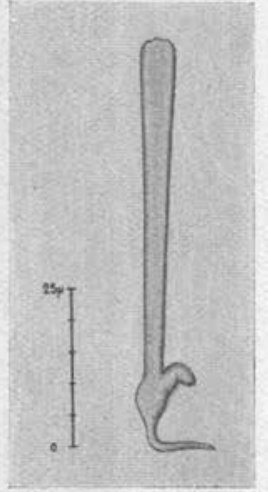

F1G. 2, - Ostobothrium leptogaster. Petit crochet. On le trouve le plus souvent au nombre de 1 à 3 exemplaires, 5 au maximum. Sa longueur est variable et très 'relative parce qu'elle dépend de la grande extensibilité de la région située entre la partie principale du corps, contenant l'appareil reproducteur, et le disque de fixation. Le plus long spécimen mesurait environ $50 \mathrm{~mm}$. Le disque, à l'aide duquel le parasite se fixe aux branchies, présente, outre les 8 ventouses munies d'un appareil chitineux compliqué, une paire de crochets plus grands et une paire de crochets médians très petits, de forme bizarre (fig. 2). La longueur des petits crochets est de 62 à $65 \mu$.

\section{Macraspis elegans Olsson}

Cet intéressant représentant de la famille des Aspidogastridés a été étudié principalement par Olsson $(1868,1870,1896)$ et par Jägerskiöld (1899). Je le trouvais assez fréquemment dans la vésicule biliaire : sur 76 chimères examinées, il y en avait 29 d'infestées, donc 38 p. cent. On trouve le plus souvent de 1 à 3 exemplaires, plus rarement davantage, 5 exemplaires au maximum. Parmi les individus adultes, à utérus rempli d'œufs, on trouvait de temps en temps des parasites jeunes sans œufs. Les dimensions des œufs, munis d'opercule, sont de $120 \times 80 \mu$, chiffres différents de ceux donnés par Olsson. Le développement du parasite reste inconnu jusqu’à présent.

\section{Calicotyle kroyeri Diesing 1850}

Ce trématode a été observé chez plusieurs espèces, appartenant à la famille des Rajidés, cependant il n'a pas été signalé comme parasite des chimères même par Olsson. Or, C. kroyeri n'est pas rare dans les environs de Bergen et le nombre des chimères infestées par lui est assez considérable. J'ai trouvé le plus souvent de 2 à 4 exemplaires dans le cloaque ou près de l'orifice cloacal. Le nombre de ces parasites pouvait aussi s'élever jusqu’à 10 exemplaires. 
Ce sont cependant les raies qui hébèrgent le plus grand nombre de ces vers. Un spécimen de Raia fullonica m'avait fourni 25 exemplaires de ce trématode.

\section{Metacercariæ}

Les métacercaires, enkystées dans le tube digestif des chimères (fig. 3), ont été observées par moi 14 fois, dans environ $15 \mathrm{p}$. cent des cas : quatre fois, il y avait 2 exemplaires, dix fois, 1 exemplaire ; six fois, c'est-à-dire le plus souvent, on rencontrait ces parasites dans le ventricule et la valvule spirale, trois fois dans la valvule spirale, quatre fois dans les autres parties du tube digestif. Il est facile de les distinguer même dans le matériel peu frais, parce qu'ils forment des épaississements sphériques de 2 à $2 \mathrm{~mm}$., 5 de diamètre. Du kyste sectionné s'échappe le trématode remuant faiblement. La longueur de la métacercaire varie de $1 \mathrm{~mm}$., 7 à $5 \mathrm{~mm} ., 8$; sa largeur est de $0 \mathrm{~mm}$., 5 à $1 \mathrm{~mm}$. sur les préparations aplaties et fixées. La ventouse ventrale est plus grande que l'orale dans les proportions de 3-2. La longueur de cette dernière ventouse est de 240 à $410 \mu$ et sa largeur, de 300 à $450 \mu$. La longueur de la ventouse ventrale est de 360 à $680 \mu$; sa largeur de 370 à $670 \mu$.

Immédiatement en arrière de la ventouse orale se trouve le pharynx musculeux, large de 120 à $210 \mu$. L'œsophage reste invisible ; la bifurcation de l'intestin commence tout près du pharynx et s'étend jusqu'à l'extrémité du corps, en formant deux branches de

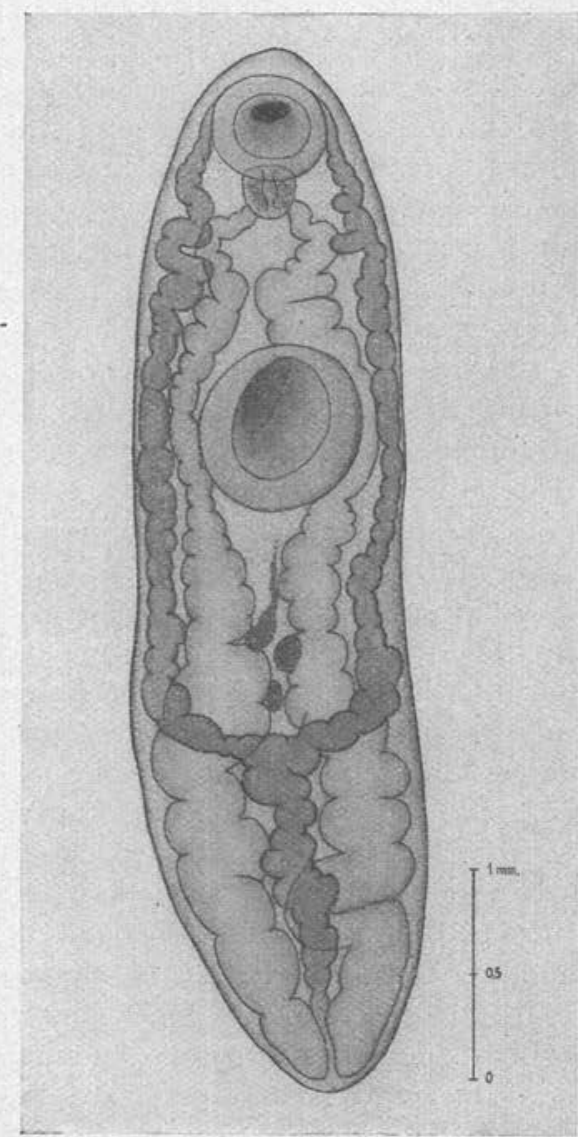

Fig. 3. - Métacercaire du tube digestif d'une chimère. 
diamètre variable dans les différentes régions du corps. On pouvait remarquer, chez un certain nombre d'exemplaires, de vagues ébauches de l'appareil reproducteur au-dessous de la ventouse ventrale. Le système excréteur a la forme de la lettre Y, dont la branche simple, placée en arrière, est moitié plus courte que la partie bifurquée, qui s'étend jusqu'à la ventouse orale. Le tégument est complètement lisse sur toute sa surface.

L'évolution des métacercaires est inconnue. D'après Olsson (1896), les chimères sont dévorées par Scymnus borealis; il est donc possible que ce requin constitue l'hôte définitif du parasite.

\section{Kyste du cœur}

J'ai souvent rencontré des kystes dans le cœur et les branchies des chimères. Ces kystes (fig. 4), de forme sphérique, à enveloppe mince et transparente, avaient généralement de 190 à $210 \mu$ de diamètre, parfois même $260 \mu$. A l'intérieur, on remarquait, avant tout, des corps irréguliers fortement réfringents. L'examen plus minutieux permit de révéler également la présence de quelques microorganismes, visibles même à travers le kyste ( 3 à 20 exemplaires). Quand on déchire l'enveloppe du kyste, il s'en échappe des larves ciliées, dont l'aspect et le mouvement rappellent, d'une façon absolue, les miracidiums (fig. 5). Elles ont de 70 à $100 \mu$ de longueur et de 40 à $50 \mu$ de largeur. L'extrémité antérieure de cette larve présente une sorte de trompe, pareille à celle qu'on trouve chez certains miracidiums.

A l'intérieur de la larve, dans la partie antérieure, on voit deux cellules, symétriquement disposées et deux corpuscules complètement transparents et très réfringents. Ces corpuscules ne disparaissent pas après le traitement par l'alcool, par l'alcool-formol, ou par le liquide de Bouin ; ils ne se colorent, ni par le carmin au borax, ni par le carmin aluné. La plus grande partie de la moitié postérieure du corps est remplie des cellules qui, par leur position et leur aspect extérieur, rappellent les cellules germinatives du miracidium. Enfin, presque à l'extrémité du corps, on trouve un certain nombre (toujours plus de 10) de corpuscules granuleux. Il est difficile de trouver leur analogie dans le miracidium.

Bien que je n'aie trouvé aucune mention relative à la présence d'un miracidium dans un kyste, j'estime néanmoins que les kystes trouvés dans le cœur et les branchies des chimères, représentent bien une forme larvaire d'un trématode inconnu jusqu'à présent. Il est possible que l'enkystement ne soit qu'une phase qui précède 
la dégénérescence complète du parasite. On trouve, en outre, simultanément, dans le cœur des chimères infestées, d'autres corps sphériques de nature cristalline. Ces corps, quand on les écrase, se désagrègent en quelques fragments irréguliers. Il m'a été impossible d'établir le rapport entre ces corps et les kystes, ou de trouver les formes intermédiaires.

A côté des vers qui sont, sans aucun doute, parasites des chimè-

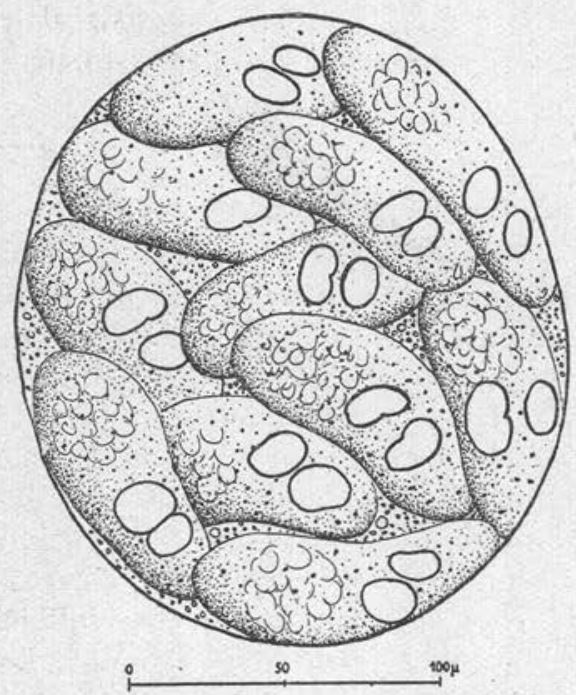

Fig. 4. - K̇yste provenant du cœur d'une chimère.

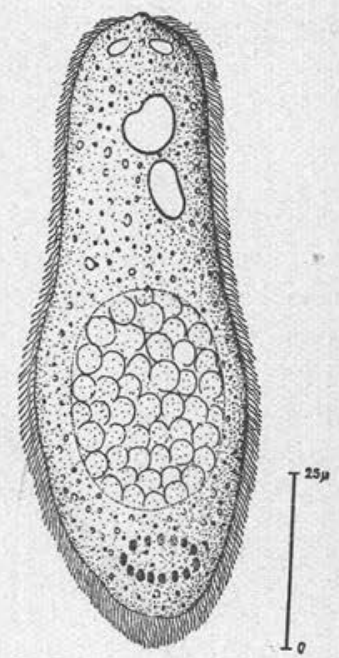

FIG. 5. - Miracidium (?) extrait d'un kyste.

res, j'en ai rencontré quelques autres, au sujet desquels je ne peux pas me prononcer avec certitude, ne sachant si les chimères sont réellement leurs véritables hôtes. Peut-être ces parasites ont-ils pénétré avec la nourriture? De toute façon, je donne sommairement leur description, uniquement dans le but d'attirer sur eux l'attention des zoologistes.

\section{Lebouria sp.}

J'ai trouvé à deux reprises (chaque fois 2 exemplaires) ce trématode, qui ressemblait à Lebouria alacris (Loos, 1906), parasite des poissons de la famille des Labridés. La longueur du ver, sur les préparations totales, était de $1 \mathrm{~mm} ., 75$; les dimensions de la ventouse 
orale étant de $300 \times 350 \mu$; celles de la ventouse ventrale de $430 \mu$ de diamètre. La longueur du pharynx était de $100 \mu$. L'œsophage était assez court. Les branches de l'intestin étaient presque invisibles ; il semble cependant qu'elles s'étendent jusqu'à l'extrémité du corps. Les testicules, ovalaires, sont disposés l'un après l'autre et un peu obliquement. Ils sont situés dans la ligne médiane du trématode. L'ovaire est placé en avant du testicule antérieur, à droite et un peu obliquement. L'utérus est petit et contient peu d'œufs, de $75 \times 45 \mu$ environ. Le pore génital s'ouvre en avant de la ventouse ventrale. Les glandes vitellogènes s'étendent depuis le pharynx jusqu’à la,région postérieure du corps.

\section{Distomum sp.}

J'ai trouvé une fois, dans l'estomac d'une chimère, deux trématodes à l'état jeune. Leur tégument était couvert d'épines, plus denses dans la partie antérieure, moins denses dans la partie pos-

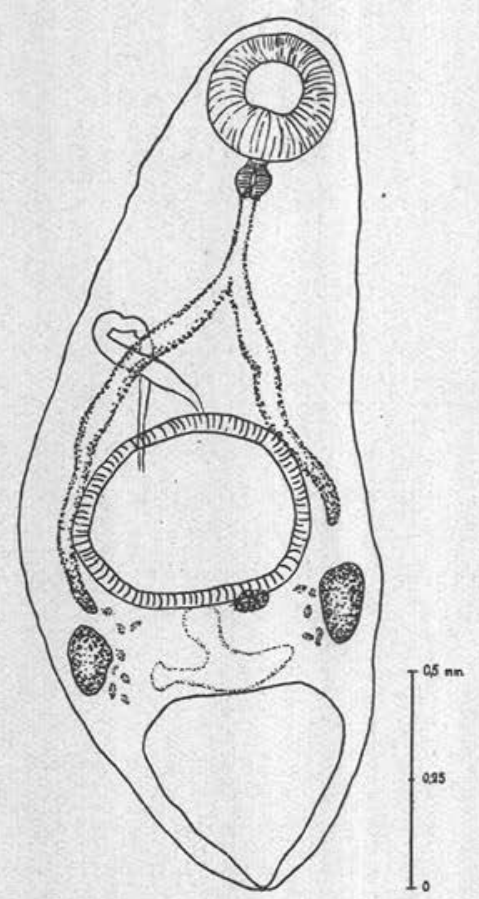

Fig, 6, - Distomum sp. térieure. La longueur du parasite, étalé (fig. 6), était de $2 \mathrm{~mm}$. La ventouse orale mesurait $280 \mu$ de diamètre, tandis que les dimensions de la ventouse ventrale étaient de $480 \times 570 \mu$. Le pharynx était petit; l'œsophage assez long. Les branches de l'intestin atteignaient le bord inférieur de la ventouse ventrale. L'un des deux spécimens présentait une branche intestinale plus courte que l'autre.

Les testicules, disposés latéralement, étaient situés au même niveau, au-dessous de la ventouse ventrale. L'ovaire était placé entre les deux testicules, plus près cependant du testicule gauche. Le pore génital s'ouvrait en avant de la ventouse ventrale. A côté des testicules, on apercevait les ébauches des glandes vitellogènes. La vésicule excrétrice était très volumineuse. 


\section{Nematoda}

Je n'ai trouvé qu'un seul nématode, long de $20 \mathrm{~mm}$. environ, dans la valvule spirale. Ne disposant pas d'un matériel suffisamment abondant, il m'est impossible de donner à son sujet des renseignements plus précis.

Avant de terminer cet exposé, je tiens à exprimer mes sincères remerciements au professeur A. Brinkmann, Directeur de la Station biologique, qui a bien voulu s'intéresser à mon travail et dont j'ai pu apprécier l’inépuisable complaisance.

\section{BIBLIOGRAPHIE}

Diesing (C. M.). - Systema helminthum. Vindobonæ, 1850.

Fuhrmann (O.). - Cestodea. W. Kükenthal Handb. d. Zoologie, II, fase. 7, 1930. Grube (Ed.). - Bemerkungen über einige Helminthen und Meerwürmer. Arch. Naturgesch., XXI, 1855.

JägerskiöLd (L. A.). - Ueber den Bau von Macrospis elegans Olss. Oefversigt Kongl. Vetenskaps-Akad. Förhandl., 1899.

LeUckart (F. S.). - Zoologische Bruchstüclee. III. Helminthologische Beiträge. Freib. (Acad. Programm.), 1842.

Looss (A.). - Zur Kenntnis der Trematodenfauna des Triester Hafens. Centralbl. f. Bakt., XXXI et XXXII, 1902, p. 115 et 637.

OLsson (P.). - Berättelse om en zoologisk resa titt Bohuslän och Skagerrack scmmaren 1868. Oefversigt Kongl. Vetenskaps-Akad. Förhandl, 1868, p. $478-479$.

- Om Entozoernas geografiske utbre dning och förekomst hos olika djur. Forhandl. ved de Skandin. Naturforskeres. Christiania, 1868-1869.

Nova genera parasitantia Copepodorum et Platyelminthium. Acta Univ. Lund., 1869-1870.

Sur Chimaera monstrosa et ses parasites. Mém. Soc. Zool. de France, IX, 1896, p. 499 .

Ruszkowski (J. S.). - Etudes sur le cycle évolutif et sur la structure des cestodes de mer. II. Sur les larves de Gyrocotyle urna (Gr. et Wagen). Bull. Ac. Pol. Sc. Cracovie, 1931-32.

Wagener (G. R.). - Ueber einen neuen in der Chimaera monstrosa gefundenen Eingeweidewurm. Müller's Arch. f. Anat. u. Phys., 1852.

Wievrejski (A.). - Zur Kenntniss des Baues von Galicotyle kroyeri Dies. Zeitschr. f. wiss. Zool., XXIX, 1877.

\section{Laboratoire de Zoologie de l'Université de Varsovie.}

